\title{
HISTIOCYTOSE LANGERHANSIENNE MULTIVISCERALE AVEC ATTEINTE AURICULAIRE BILATERALE A PROPOS D'UNE OBSERVATION
}

\author{
I. LAHMAR, B. MAHJOUB ${ }^{*}$, S. JERBI ${ }^{* *}$, A. BERKAOUI, W. BAHRI, K. MIGHRI, \\ M. T. SFAR ${ }^{*}$, N. DRISS. \\ SERVICE D'ORL \\ * SERVICE DE PEDIATRIE \\ ** SERVICE D'IMAGERIE MEDICALE \\ HOPITAL DE MAHDIA
}

\begin{abstract}
L'histiocytose langerhansienne multi-viscérale est une prolifération clonale des cellules de Langerhans, touchant plusieurs organes. Cette entité se voit surtout chez l'enfant.

Dans ce travail, nous rappelons les aspects cliniques avec la fréquence d'atteinte oto-rhino-laryngologique, ainsi que les moyens de diagnostic et le traitement de cette affection rare.

Nous présentons le cas d'un enfant âgé de 2 ans qui a été hospitalisé pour une pneumopathie interstitielle, associée à une otorrhée bilatérale.

L'examen a montré un comblement des 2 conduits auditifs externes et des lésions cutanées squameuses.

La biopsie a conclu à une histiocytose langerhansienne. Malgré la chimiothérapie, l'enfant est décédé après 11 mois.

Mots-clés : Histiocytose langerhansienne, atteinte auriculaire.
\end{abstract}

Systemic langerhans' cell histiocytosis is a clonal proliferation of langerhans cells, affecting many organs. This entity affects children.

We study clinical manifestations, diagnosis modalities and treatment of this rare disease.

We present a case of 2 years old boy which presented bilateral pneumopathy with bilateral otorrhea.

Patient had bilateral external ear granulations and skin squamous lesions. Histopathologic study after biopsy concluded to a Langerhans' cell histiocytosis. Although chemotherapy patient had died after 11 months.

In this report, we study clinical manifestations and therapeutic modalities of this rare affection.

Keywords : Langerhans' cell histiocytosis, ear lesions.

\section{INTRODUCTION}

L'histiocytose multi-viscérale est une affection rare et grave qui touche l'enfant.

Elle est caractérisée par son polymorphisme clinique qui peut parfois retarder le diagnostic. Sa gravité réside dans la possibilité d'atteinte de certains organes dits «à risque» et donc la possibilité de décès ou de séquelles.

\section{OBSERVATION}

II s 'agit d'un enfant âgé de 2 ans, hospitalisé au service de Pédiatrie en janvier 2007 pour dyspnée avec toux grasse et lésions vésiculo-squameuses du cuir chevelu et du tronc. Par ailleurs, il avait une otorrhée bilatérale.

L'examen physique a montré, outre l'atteinte pulmonaire, une hépato splénomégalie ainsi que du tissu polypoide comblant les 2 conduits auditifs externes.

Les explorations para-cliniques ont montré :

- des lacunes osseuses à la radiographie du crâne (figure 1).

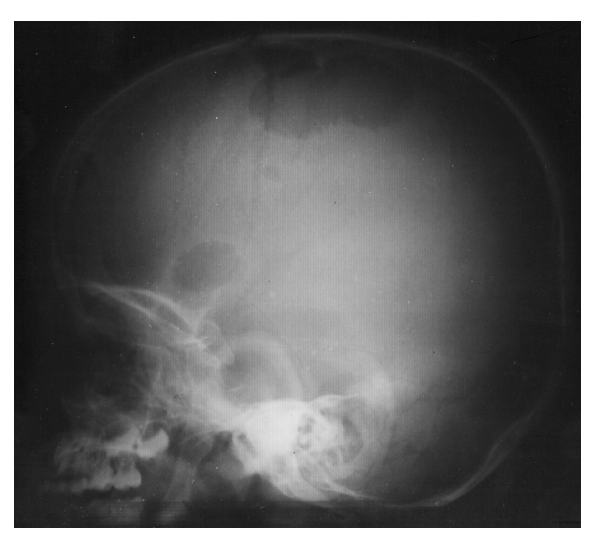

Fig. 1 : Lacunes osseuses à la radiographie du crâne

- des opacités réticulo-nodulaires bilatérales ainsi que des adénopathies médiastinales à la radiographie et au scanner du thorax.

Le scanner des rochers a montré un comblement de 
l'oreille moyenne et externe des deux cotés (figures 2 et 3 ).
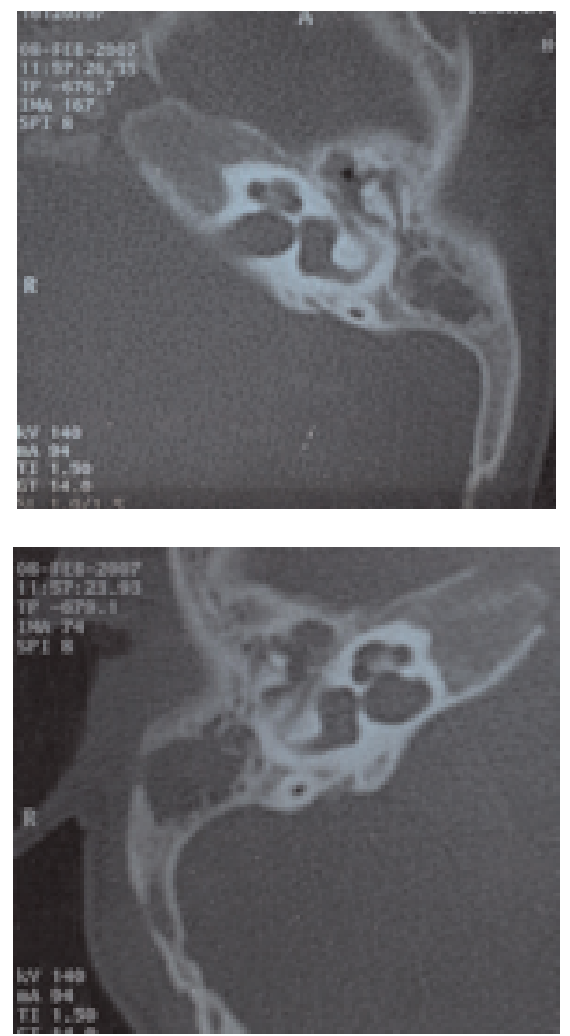

Figures 2 et 3 : Comblement bilatéral de l'oreille moyenne au scanner des rochers

Les prélèvements histologiques ont tous conclu à une histiocytose langerhansienne (Biopsies cutanée, hépatique et auriculaire) (Figure 4).

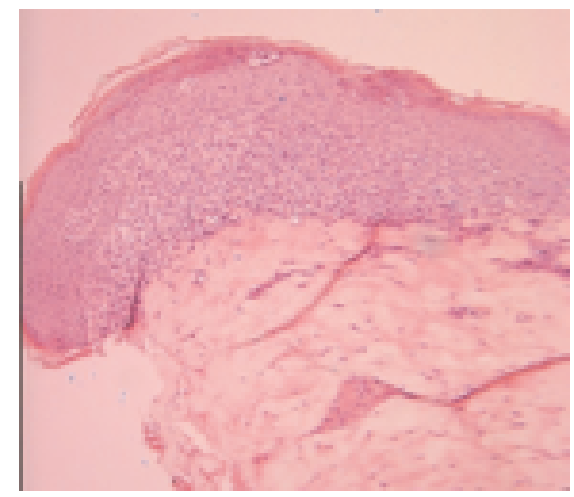

Figure 4 : Aspect histologique (biopsie cutanée) : Prolifération de cellules de langerhans

Le diagnostic d'une histiocytose langerhansienne de groupe 3 (à haut risque) a été donc retenu.

Trois cures de Vinblastine et Prednisone de 6 semaines chacune ont été réalisées, associées à un traitement de la cholestase par l' Ursolvan*.

Malgré ce traitement la maladie est restée évolutive et le patient est décédé par décompensation respiratoire et ce 11 mois après.

\section{DISCUSSION}

L'histiocytose langerhansienne $(\mathrm{HL})$, autrefois appelée histiocytose $X$ est une maladie rare à grande variabilité dans sa présentation clinique et les organes qu'elle touche (1). Son polymorphisme clinique rend son diagnostic parfois difficile (2). Identifiée il y a plus d'un siècle, c'est une prolifération clonale d'histiocytes proches des cellules présentatrices d'antigènes de la peau, les cellules de Langerhans (3). Le terme d'histiocytose $X$ remonte à l'année 1953 quand Lichtenstein a regroupé les différents syndromes réalisés par la maladie (in 4).

\section{* Physiopathogénie}

L'HL est une prolifération de cellules dendritiques de Langerhans. Compte tenu du rôle de ces cellules dans les réponses immunitaires, on pourrait s'attendre alors à des déficits immunitaires manifestés par une susceptibilité à certaines infections. Cependant, il n'y a pas encore de données, chez l'homme, concernant ce sujet (4). Quelle que soit l'expression clinique de la maladie, l'histiocytose reste une prolifération clonale et les anomalies du caryotype y sont exceptionnelles (3).

\section{* Epidémiologie}

Peu d'études ont été présentées concernant ce sujet. Cependant il est connu que les formes multi-viscérales sont l'apanage du petit nourrisson, contrairement aux granulomes à éosinophiles du grand enfant et de l'adulte $(3,5)$.

L'incidence de la maladie est de 4-5 cas/100 000 personnes/ an .

L'âge moyen est de 1 à 3 ans avec une prédominance masculine (6).

\section{* Etude clinique}

L'HL est connue par son polymorphisme clinique $(1,3,4)$. Peu d'études ont été avancées quant à l'atteinte primitive de la tête et du cou (6). Cette atteinte, bien que parfois complexe, reste elle aussi dans le cadre des atteintes « mono focales » tout comme l'atteinte des os longs (6).

L'atteinte de la tête et du cou varie entre $67 \%$ et $82 \%$ dans la littérature ; la localisation cervicale pure entre 8 et $26 \%$ (in 6).

Dans une série de 49 patients, Bernstrand (7) a rapporté 10 cas d'atteinte multi-viscérale et un dysfonctionnement d'organe dans uniquement 6 cas. Une atteinte auriculaire a été notée dans $16 \%$ des cas au moment du diagnostic et dans $20 \%$ des cas lors de l'évolution de la maladie. Cette atteinte auriculaire est manifestée par une otorrhée et un comblement polyploïde du conduit auditif externe. 
Les organes touchés par la maladie, outre la tête et le cou, peuvent être la peau (aspect en maillot de corps), les os longs, l'appareil digestif. L'atteinte d'autres organes rend péjoratif le pronostic : foie, poumon, moelle osseuse et rate (3).

Pour cela, une classification a été avancée par l'histiocyte society (3) :

* groupe 1 : atteinte d'un seul organe

* groupe 2 : âge supérieur ou égal à 2 ans avec atteinte pluri focale à l'exclusion d'une atteinte des organes « à risque » (foie, poumon, rate, moelle osseuse)

* groupe 3 : maladie multifocale avec atteinte d'au moins un organe « à risque »; ou âge inférieur à 2 ans

\section{* Diagnostic}

Le diagnostic de la maladie est histo-pathologique. II s'agit d'une prolifération de cellules de Langerhans, ne présentant aucun critère de malignité.

La lésion histologique de base est un granulome fait d'amas d'histiocytes associés à des lymphocytes (3). L'imuno-histo-chimie montr une positivité au CD1a (5). En microscopie électronique, un aspect pathognomonique de la maladie est retrouvé : les granules de Birbeck de structure penta lamellaire ovale, possédant une striation périodique (image de «fermeture éclair ») $(3,6)$

\section{* Traitement et pronostic}

L'H.L. dans sa forme multi viscérale pose encore un grand problème thérapeutique. Ceci est expliqué par la grande variété des organes atteints ainsi que la rareté de la maladie elle-même $(3,8)$

Le premier protocole thérapeutique « DAL-HX 83-90 » est basé sur le prednisone ( 6 semaines) associé à la vinblastine et au VP16, puis un traitement par le purinethol*. Le deuxième protocole «LCH1 » (1991-1995) a montré une efficacité comparable de la vinblastine et du VP16 associés aux corticoïdes et au purinethol. Le dernier protocole " LCH2 » (1996-2000) a éliminé le VP16 du fait de sa toxicité et le risque d'association néoplasie / histiocytose
(3)

Malgré les différents protocoles thérapeutiques, l'histiocytose multi viscérale reste de mauvais pronostic (6). La gravité de cette maladie réside dans la possibilité d'atteinte des organes à risque et donc soit dans les décès soit dans les séquelles avec dysfonction d'organe (3).

\section{CONCLUSION}

Plus rare que la forme mono focale, l'HL multi viscérale présente, outre les problèmes diagnostiques du fait de son polymorphisme clinique, des difficultés thérapeutiques.

L'atteinte de la tête et du cou y reste élevée et son pronostic est mauvais, conditionné par l'âge et l'atteinte de certains organes dits " à risque ».

\section{REFERENCES}

1- Wei J.,Ruchmann L., Treatment of primary .Head and neck Langerhans cell histiocytosis in children Otolaryngology-Head and Neck Surgery.2005; 184 2- Calming U, Henter JI Elevated erythrocyte sedimentation rate and thrombocytosis as possible indicators of active disease in Langerhans'cell histiocytosis. Acta Paediatr 1998 ; 87 : 1085-7 ;

3- Stéphan JL. Histiocytoses langerhansiennes et non langerhansiennes Archives de pédiatrie $2002 ; 9: 934-941$

4- Geissmann F., Thomas C. Données actuelles sur la clinique la physiopathologie et le traitement de l'histiocytose langerhansiennne (histiocytose X). Arch pediatr $1999 ; 6$ (I2) : 414-6
5- Ouesleti Z., Hassen C., Haffouz S et al. Histiocytose X de localisation cervicofaciale Journal tunisien d'ORL. 2003;10 : 29-32

6- Buchmann L., Emami A, Wei JL. Primary head and neck langerhans cell histiocytosis in children Otolaryngology-Head and Neck Surgery 2006; 135: 312317

7- Bernstand C. , stands ted B. , Ahstour L. Long-tern follow-up of langerhans cell histiocytosis : 39 years experience at a single center. Acta paediatrica, 2005, 94: 1073-1084

8- Mahmoud H. H, Wang W. C., Murphy S B Cyclosporine therapy of advanced Langerhans cell histiocytosis Blood, 1991; 77(4): 721-725 